\title{
Hybrid method for solving the non smooth cost function economic dispatch problem
}

\author{
Wanchai Khamsen ${ }^{1}$, Chiraphon Takeang ${ }^{2}$, Patiphat Aunban ${ }^{3}$ \\ ${ }^{1,2}$ Department of Electrical Engineering, Rajamangala University of Technology Lanna, Thailand \\ ${ }^{3}$ Chiangmai Municipality, Thailand
}

\begin{tabular}{l}
\hline Article Info \\
\hline Article history: \\
Received Jul 9, 2018 \\
Revised Aug 29, 2019 \\
Accepted Sep 27, 2019 \\
\hline
\end{tabular}

Keywords:

Economic dispatch

Hybrid method

Optimization

\begin{abstract}
This article is focused on hybrid method for solving the non-smooth cost function economic dispatch problem. The techniques were divided into two parts according to: the incremental cost rates are used to find the initial solution and bee colony optimization is used to find the optimal solution. The constraints of economic dispatch are power losses, load demand and practical operation constraints of generators. To verify the performance of the proposed algorithm, it is operated by the simulation on the MATLAB program and tests three case studies; three, six and thirteen generator units which compared to particle swarm optimization, cuckoo search algorithm, bat algorithm, firefly algorithm and bee colony optimization. The results show that the proposed algorithm is able to obtain higher quality solution efficiently than the others methods.
\end{abstract}

Copyright $(0) 2020$ Institute of Advanced Engineering and Science. All rights reserved.

\section{Corresponding Author:}

Wanchai Khamsen,

Department of Electrical Engineering,

Rajamangala University of Technology Lanna,

200 Moo17 Pichai Subdistrict Mueang District Lampang Province, 52000, Thailand.

Email: wanchai_kh@rmutl.ac.th

\section{INTRODUCTION}

The electricity power generation enough with the demand. It is an important for security and reliability of electrical power system. Fuel is a factor that affects the production of electricity. It requires a production plan to minimize the total fuel cost of electrical power generation which satisfied constraints. That is the economic dispatch problem (ED). The objective function of economic dispatch is to minimize the total fuel cost of power plant which satisfied the constraints of load demand and power loss. The fuel cost function of electrical power generation divided into two types. The first type is smooth cost function represented by a single quadratic function, under the assumption that the incremental cost curves of the power plant are increasing piecewise linear functions. The second type is non-smooth cost function represented higher order nonlinear function and discontinuities which complex and non-convex characteristics with many constraints. While, most power plants are non-smooth cost function. The power output of the generator is controlled by multiple valves [1]. When steam admission valves in thermal units are first opened, a losses occurs rapidly. This is the valve-point loading characteristics. The economic dispatch problem with valve-point effects is difficulty of finding the global or near optimum.

There are many methods of meta heuristic optimization techniques to solving the non-smooth cost economic dispatch problem such as simulated annealing (SA) [2-5], genetic algorithm (GA) [6-7] and tabu search (TS) [8-9], particle swarm optimization (PSO) [10-13], ant colony optimization (ACO) [14-17], cuckoo search algorithm (CSA) [18-20], bat algorithm (BAT) [21-22], firefly algorithm (FFA) [23-24] and bee colony optimization (BCO) [25-29]. There are probabilistic heuristic algorithms which have been successfully used to solve the economic problem. While, BCO is very simple and robust stochastic optimization algorithm. The solution quality and computational efficiency of BCO is better than other 
algorithm. However, the initial population of all meta heuristic optimization techniques are obtained randomly, as a result more time to computational efficiency. That is the problem of this article.

The goal of this paper is to develop the hybrid method for solving the ED problem with non-smooth cost functions that good solution quality and computational efficiency. The incremental costs rates are used to find the initial solution for reduce the scope of the search and BCO to finding the global or near optimum around the initial solution. The constraints of economic dispatch are power losses, load demand and practical operation constraints of generators. Results from previous methods in terms of solution quality and computational efficiency are compared in this paper.

\section{RESEARCH METHOD}

The objective of the economic dispatch problem is to minimize the total generation cost of the individual dispatchable generating power units that satisfying the constraints.

\subsection{Objective function}

The objective function of ED problem can be formulated as a quadratic cost function:

$$
\text { Minimize }: T C=\sum_{i=1}^{N} F_{i}\left(P_{i}\right)=a_{i}+b_{i} P_{i}+c_{i} P_{i}^{2}+\left|e_{i} \times \sin \left(f_{i} \times\left(P_{i, \min }-P_{i}\right)\right)\right|
$$

where $T C$ is the total generation cost; $N$ is the number of generating units; $F_{i}\left(P_{i}\right)$ is the total fuel cost of generation; $P$ is the power output of the $i^{t h}$ generator and $a_{i}, b_{i}, c_{i}, e_{i}$ and $f_{i}$ are the cost coefficient of the $i^{t h}$ generator.

\subsection{Constrains}

The objective function represented in (1) is subject to the following equality and inequality constraints of the ED problems.

\subsubsection{Power balance constraint}

The sum of power output of all generator units must be equal to the sum of the total power demand and total power transmission losses as given below.

$$
\sum_{i=1}^{N}\left(P_{i}\right)=P_{D}+P_{l o s s}
$$

where $P_{D}$ and $P_{\text {loss }}$ are the total power demand and total power transmission losses respectively. The transmission losses are expressed as a function of the real power and $B$ coefficient matrix as given below.

$$
P_{\text {loss }}=\sum_{i=1}^{N} \sum_{j=1}^{N} P_{i} B_{i j} P_{j}+\sum_{j=1}^{N} B_{i o} P_{i}+B_{00}
$$

where $B_{00}, B_{i 0}$ and $B_{i j}$ are the loss coefficient of the transmission line that assumed to be constant under the normal operating condition.

\subsubsection{Generator rating constraint}

The power output of each generator units must be operate within lower and upper operating limit which defined as:

$$
P_{i, \min } \leq P_{i} \leq P_{i, \max }
$$

where $P_{i, \min }$ and $P_{i, \max }$ are the minimum and maximum power output of the $i^{\text {th }}$ generator unit. 


\subsection{Hybrid method (HBCO)}

In this section, a new approach to implement the hybrid algorithm will be described in solving the ED problems. The hybrid algorithm techniques combination of two methods. The first one is the incremental cost rates $(\lambda)$ which used to find the initial solution. The approach could provide local optimum solution. The second method is used BCO to find the optimal solution of initial neighborhood. Therefore, hybrid algorithm is obtained the incremental cost rates and BCO (HBCO). The constraints of ED are power losses, load demand and practical operation constraints of generators. To verify the performance of the proposed algorithm, it is operated by the simulation on the MATLAB program and tests three case studies; three, six and thirteen generator units with losses and without transmission losses. The process of the hybrid method is summarized as follows:

Step 1: Specify the HBCO parameters as shown in Table 1.

Step 2: Calculate the incremental cost rates $(\lambda)$ using the following:

$$
\lambda=\frac{P_{D}+\sum_{i=1}^{N} \frac{b_{i}}{c_{i}}}{\sum_{i=1}^{N} \frac{1}{c_{i}}}
$$

Step 3: Calculate the power output of the $i^{\text {th }}$ generator $\left(P_{i}\right)$ with incremental cost rates that the initial solutions as following:

$$
P_{i}=\frac{\lambda-b_{i}}{c_{i}}
$$

Step 4: Find boundary of the power output of the $i^{\text {th }}$ generator using the following:

$$
P_{i, \text { lower }}=P_{i}(1-\text { rank })
$$

and

$$
P_{i, \text { upper }}=P_{i}(1+\text { rank })
$$

where $P_{i, \text { lower }}$ and $P_{i, \text { upper }}$ are the minimum and maximum power output of the $i^{\text {th }}$ generator unit; rank is rank size of power output generation.

Step 5: Create the populations $(N)$ of the power output of the $i^{\text {th }}$ generator that satisfied the constraints can be expresses as:

$$
P_{i}=P_{i, \text { lower }}+\left(\left(P_{i, \text { upper }}-P_{i, \text { lower }}\right) \cdot \operatorname{rand}(0,1)\right)
$$

Step 6: Evaluate the fitness value of the populations and arrange the fitness in ascending order.

Step 7: Select $S$ best solutions for the neighborhood search and separate the $S$ best solutions into two groups $(E, S-E)$.

Step 8: Determine the size of neighborhood for each best solution. Note that neighborhood sizes are equal to $N E$ for solution group $E$ and $N O$ for solution group $(S-E)$.

Step 9: Generate solutions around the selected solutions within the neighborhood sizes $(N E, N O)$ and evaluate the fitness value from each patch. Then, select the best solution from each patch.

Step 10: Check the stopping criterion. If no, increase the iteration.

Step 11: Assign the new population $(N-S)$ to generate new power output of the $i^{\text {th }}$ generator. Then, return to Step 5. 
Table 1. The HBCO parameters

\begin{tabular}{lc}
\hline Parameters & Number \\
\hline Population size $(N)$ & 20 \\
Number of selected sites $(S)$ & 14 \\
Number of best sites $(E)$ & 10 \\
Number of bees around best sites $(N E)$ & 20 \\
Number of bees around other sites $(N O)$ & 10 \\
Rank size (rank) & 0.2 \\
\hline
\end{tabular}

\section{RESULTS AND ANALYSIS}

The aim of this paper is to develop the hybrid method for solving the ED problem with non-smooth cost functions that good solution quality and computational efficiency. In this study, the three difference test cases are considered for verifying the effectiveness of the proposed approach.

\subsection{Test case 1: three units system}

This case study is the simple system with three generators and a total load demand of 850 MW. The system data is shown in Table 2. The simulation results from the proposed HBCO, PSO, CSA, BAT, FFA, and BSO algorithms are compared in Table 3. The results indicate that the proposed HBCO succeeds in finding the best solution of total generation cost. The convergence characteristics of the proposed HBCO in comparison with BCO methods are shown in Figure 1. Clearly, the HBCO converges to the optimal solution faster than $\mathrm{BCO}$ methods.

Table 2. Generator data for case 1

\begin{tabular}{cccc}
\hline Unit No. & 1 & 2 & 3 \\
\hline$a_{i}$ & 0.001562 & 0.00194 & 0.00482 \\
$b_{i}$ & 7.92 & 7.85 & 7.97 \\
$c_{i}$ & 561 & 310 & 78 \\
$e_{i}$ & 300 & 200 & 150 \\
$f_{i}$ & 0.315 & 0.42 & 0.63 \\
$P_{i, \max }$ & 600 & 400 & 200 \\
$P_{i, \min }$ & 100 & 100 & 50 \\
\hline
\end{tabular}

Table 3. Comparison of the best results of each method for case 1

\begin{tabular}{ccccccc}
\hline Unit No. & PSO [13] & CSA [20] & BAT [21] & FFA [24] & BCO [27] & HBCO \\
\hline 1 & 481 & 498.62 & 384.423 & 384.423 & 300.266 & 386.083 \\
2 & 279 & 101.319 & 151.643 & 151.643 & 149.734 & 339.361 \\
3 & 90 & 250.061 & 313.934 & 313.934 & 400.000 & 125.946 \\
Total $P(\mathrm{MW})$ & 850 & 850 & 850 & 850 & 850.000 & 851.390 \\
Total Cost $(\$ / \mathrm{hr})$ & 8217 & 8248.2 & 8253.1 & 8253.1 & 8234.07 & 8210 \\
\hline
\end{tabular}

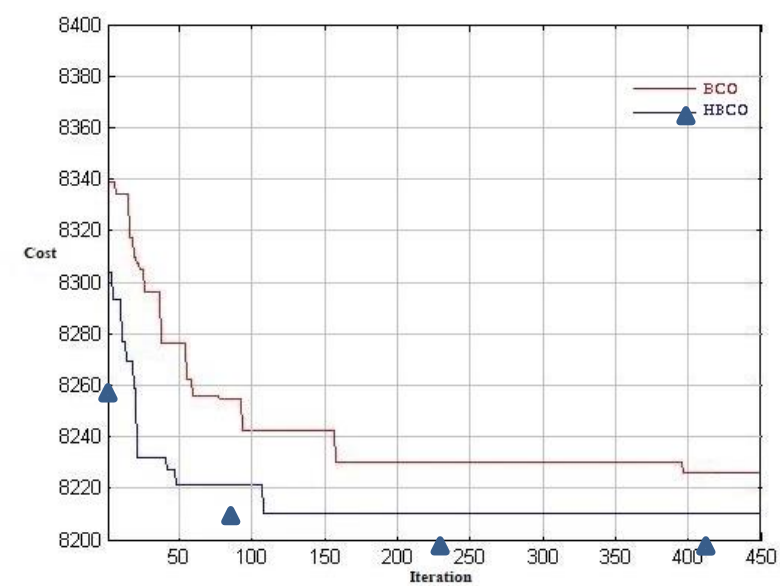

Figure 1. Solution convergence of proposed method (HBCO) and BCO for case 1 


\subsection{Test case 2: six units system}

In this case, the system consists of six generators. The characteristic of all thermal generating units with valve point effect are given in Table 4 . The total load demand is $1263 \mathrm{MW}$ and loss coefficients matrix as follow:

$$
\begin{aligned}
& B_{i j}=1 \times 10^{-5}\left[\begin{array}{cccccc}
0.17 & 1.2 & 0.7 & -0.1 & -0.5 & -0.2 \\
1.2 & 1.4 & 0.9 & 0.1 & -0.6 & -0.1 \\
0.7 & 0.9 & 3.1 & 0 & -1.0 & -0.6 \\
-0.1 & 0.1 & 0 & 2.4 & -0.6 & -0.8 \\
-0.5 & -0.6 & -1 & -0.6 & 12.9 & -0.2 \\
-0.2 & -0.1 & -0.6 & -0.8 & -0.2 & 15.0
\end{array}\right] \\
& B_{0 i}=1 \times 10^{-5}\left[\begin{array}{llllll}
-3.908 & -1.297 & 7.047 & 0.591 & 2.161 & -6.635
\end{array}\right] \quad B_{00}=\left[\begin{array}{llll}
0.056
\end{array}\right]
\end{aligned}
$$

Table 5 show the summarized result of all the existing algorithms along with proposed method (HBCO) for test case 2. The simulation results from the proposed HBCO, MPSO, CSA, MABC, and IASFLA algorithms are compared. The results indicate that HBCO can provide a better solution than the other approaches in total generation cost and convergence efficiently. The convergence characteristics of the proposed $\mathrm{HBCO}$ in comparison with $\mathrm{BCO}$ methods are shown in Figure 2.

Table 4. Generator data for case 2

\begin{tabular}{ccccccc}
\hline Unit No. & 1 & 2 & 3 & 4 & 5 & 6 \\
\hline$a_{i}$ & 0.0070 & 0.0095 & 0.0090 & 0.0090 & 0.0080 & 0.0075 \\
$b_{i}$ & 7.0 & 10 & 8.5 & 11 & 10.5 & 12 \\
$c_{i}$ & 240 & 200 & 220 & 200 & 220 & 190 \\
$e_{i}$ & 300 & 200 & 400 & 159 & 150 & 150 \\
$f_{i}$ & 0.035 & 0.042 & 0.042 & 0.063 & 0.063 & 0.063 \\
$P_{i, \text { max }}$ & 500 & 200 & 300 & 150 & 200 & 120 \\
$P_{i, \text { min }}$ & 100 & 50 & 80 & 50 & 50 & 50 \\
\hline
\end{tabular}

Table 5. Comparison of the best results of each method for case 2

\begin{tabular}{cccccc}
\hline Unit No. & MPSO [12] & CSA [18] & MABC [26] & IASFLA [30] & HBCO \\
\hline 1 & 447.187 & 447.4768 & 449.839 & 446.721 & 470.31 \\
2 & 173.506 & 173.223 & 173.380 & 175.777 & 151.839 \\
3 & 260.955 & 263.379 & 257.037 & 264.612 & 268.437 \\
4 & 144.058 & 138.952 & 142.346 & 140.286 & 105.79 \\
5 & 163.216 & 165.412 & 161.724 & 160.934 & 177.008 \\
6 & 86.293 & 87.002 & 90.58 & 87.100 & 99.533 \\
Total P(MW) & 1275.22 & 1275.45 & 1274.91 & 1275.43 & 1272.92 \\
Total Cost (\$/hr) & 15,441 & 15,443 & 15,438 & 15,442 & 15,430 \\
Power losses (MW) & 12.216 & 12.447 & 11.907 & 12.33 & 9.74 \\
\hline
\end{tabular}

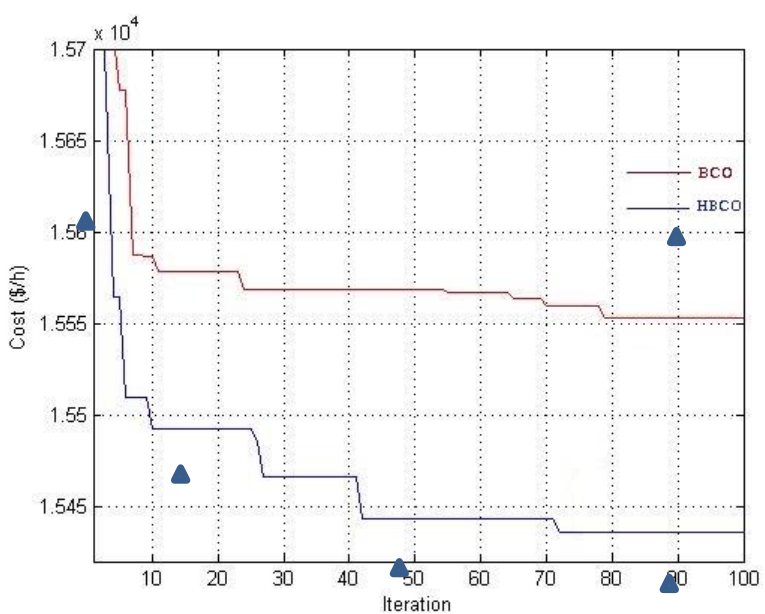

Figure 2. Solution convergence of proposed method (HBCO) and BCO for case 2 


\subsection{Test case 3: thirteen units system}

In this case, the system consists of 13 generating units. The characteristic of all thermal generating units with valve point effect are given in Table 6 . In order to validate the proposed HBCO method, it is tested with 13-unit system having non-convex solution spaces and total load demands of $1800 \mathrm{MW}$. Table 7 shows the comparison of results with different methodologies. The results indicate that optimal value of fuel cost obtained by HBCO algorithm is much less that FAPSO-VDE, CSA, FA and ABC. The convergence characteristics of the proposed $\mathrm{HBCO}$ in comparison with BCO methods are shown in Figure 3 Clearly, the $\mathrm{HBCO}$ converges to the optimal solution faster than BCO methods.

Table 6. Generator data for case 3

\begin{tabular}{cccccccc}
\hline Unit No. & $a_{i}$ & $b_{i}$ & $c_{i}$ & $e_{i}$ & $f_{i}$ & $P_{i, \max }$ & $P_{i, \min }$ \\
\hline 1 & 0.00028 & 8.10 & 550 & 300 & 0.035 & 680 & 0 \\
2 & 0.00056 & 8.10 & 309 & 200 & 0.042 & 360 & 0 \\
3 & 0.00056 & 8.10 & 307 & 200 & 0.042 & 360 & 0 \\
4 & 0.00324 & 7.74 & 240 & 150 & 0.063 & 180 & 60 \\
5 & 0.00324 & 7.74 & 240 & 150 & 0.063 & 180 & 60 \\
6 & 0.00324 & 7.74 & 240 & 150 & 0.063 & 180 & 60 \\
7 & 0.00324 & 7.74 & 240 & 150 & 0.063 & 180 & 60 \\
8 & 0.00324 & 7.74 & 240 & 150 & 0.063 & 180 & 60 \\
9 & 0.00324 & 7.74 & 240 & 150 & 0.063 & 180 & 60 \\
10 & 0.00284 & 8.60 & 120 & 100 & 0.084 & 120 & 40 \\
11 & 0.00284 & 8.60 & 120 & 100 & 0.084 & 120 & 40 \\
12 & 0.00284 & 8.60 & 120 & 100 & 0.084 & 120 & 55 \\
13 & 0.00284 & 8.60 & 120 & 100 & 0.084 & 120 & 55 \\
\hline
\end{tabular}

Table 7. Comparison of the best results of each method for case 3

\begin{tabular}{cccccc}
\hline Unit No. & FAPSO-VDE [11] & CSA [20] & FA [23] & ABC [27] & HBCO \\
\hline 1 & 628.319 & 369.055 & 628.319 & 628.277 & 502.641 \\
2 & 227.749 & 227.735 & 149.599 & 148.882 & 326.124 \\
3 & 149.599 & 62.177 & 222.749 & 223.616 & 251.768 \\
4 & 60 & 108.771 & 109.867 & 60 & 88.218 \\
5 & 109.867 & 107.438 & 109.867 & 109.853 & 88.263 \\
6 & 109.867 & 120 & 109.867 & 109.84 & 88.27 \\
7 & 109.867 & 163.739 & 109.867 & 109.861 & 88.24 \\
8 & 109.867 & 156.243 & 60 & 109.855 & 88.162 \\
9 & 109.867 & 138.671 & 109.867 & 109.826 & 88.165 \\
10 & 40 & 108.807 & 40 & 40 & 40 \\
11 & 40 & 115.757 & 40 & 40 & 40 \\
12 & 55 & 62.259 & 55 & 55 & 40 \\
13 & 55 & 59.349 & 55 & 55 & 55 \\
Total $P(\mathrm{MW})$ & 1800 & 1800 & 1800 & 1800 & 1800 \\
Total Cost $(\$ / \mathrm{hr})$ & 17963.82 & 18809 & $17,963.83$ & 17962.43 & $17,946.55$ \\
\hline
\end{tabular}

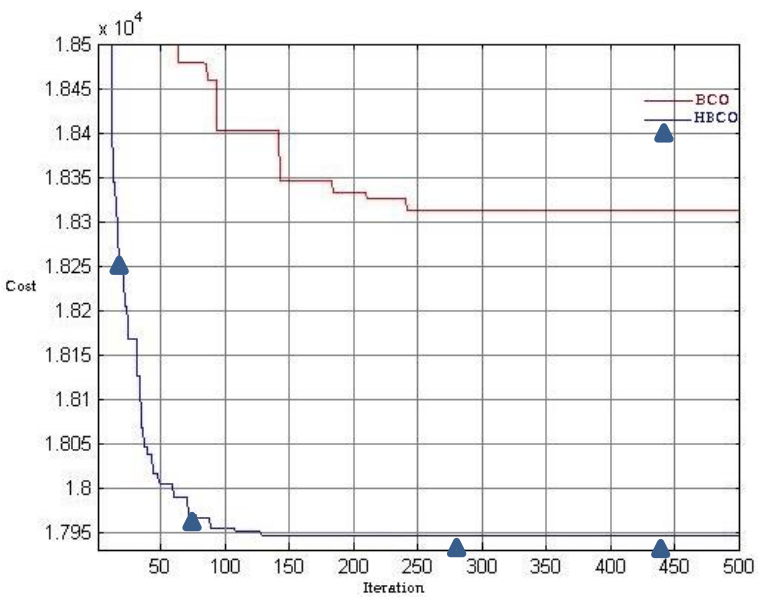

Figure 3. Solution convergence of proposed method (HBCO) and BCO for case 3 


\section{CONCLUSION}

This paper proposes a methodology for solving the ED problem with non-smooth cost functions using hybrid method with taking various generator constraints. Three case systems are tested evaluates the performance proposed approach. The HBCO shown that algorithm is robust and can provide good solution quality and computational efficiency. The studied results confirm the HBCO proposed approach are indeed capable of obtaining higher quality solution, computation time and convergence characteristic in comparison with other method. The aim of this paper is to develop the hybrid method for solving the ED problem with non-smooth cost functions that good solution quality and computational efficiency.

\section{ACKNOWLEDGEMENTS}

The authors would like to thanks Prof. Dr. Ian Warrington for valuable suggestion.

\section{REFERENCES}

[1] A. Jabr, et al., "A Homogeneous Linear Programming Algorithm for the Security Constrained Economic Dispatch Problem," IEEE Trans. Power Systems, vol. 15, pp. 930-7, 2000.

[2] J. Sasikala and M. Ramaswamy, "Optimal $\lambda$ Based Economic Emission Dispatch using Simulated Annealing," International Journal of Computer Applications, vol. 1, pp. 55-63, 2010.

[3] H. Hardiansyah, et al., "An Efficient Simulated Annealing Algorithm for Economic Load Dispatch Problems," Telecommunication, Computing, Electronics and Control, vol. 11, pp. 37-46, 2013.

[4] Z. Ismail, et al., "Simulated Annealing Optimization for Multi-objective Economic Dispatch Solution," Leonardo Journal of Sciences, vol. 25, pp. 43-56, 2014.

[5] M. Basu, "A Simulated Annealing-Based Goal-Attainment Method for Economic Emission Load Dispatch of Fixed Head Hydrothermal Power Systems," Electrical Power and Energy Systems, vol. 27, pp. 147-153, 2005.

[6] C. L. Chiang, "Improved Genetic Algorithm for Power Economic Dispatch of Units with Valve-Point Effects and Multiple Fuels," IEEE Transactions on Power Systems, vol. 20, pp.1690-1699, 2005.

[7] S. Dinu, et al., "Environmental Economic Dispatch Optimization using a Modified Genetic Algorithm," International Journal of Computer Applications, pp. 7-14, 2011.

[8] K. Senthil and K. Manikandan, "Economic Thermal Power Dispatch with Emission Constraint and Valve Point Effect Loading Using Improved Tabu Search Algorithm," International Journal of Computer Application, pp. 6-11, 2010.

[9] W. Sa-ngiamvibool, et al., "Multiple Tabu Search Algorithm for Economic Dispatch Problem Considering ValvePoint Effects," Electrical Power and Energy Systems, vol. 33, pp. 846-854, 2011.

[10] M. Neyestani, et al., "A Modified Particle Swarm Optimization for Economic Dispatch with Non-Smooth Cost Functions," Engineering Applications of Artificial Intelligence, vol. 23, pp. 1121-1126, 2010.

[11] T. Niknam, et al., "A Novel Hybrid Particle Swarm Optimization for Economic Dispatch with Valve-Point Loading Effects," Energy Conversion and Management, vol. 52, pp. 1800-1809, 2011.

[12] Hardiansyah, "A Modified Particle Swarm Optimization Technique for Economic Load Dispatch with Valve-Point Effect," I.J. Intelligent Systems and Applications, vol. 7, pp. 32-41, 2013.

[13] P. S. Bhullar and J. K. Dhami, "Particle Swarm Optimization Based Economic Load Dispatch with Valve Point Loading," International Journal of Engineering Research \& Technology, pp. 1064-1070, 2015.

[14] S. Pothiya, et al., "Ant Colony Optimisation for Economic Dispatch Problem with Non-Smooth Cost Functions," Electrical Power and Energy Systems, vol. 32, pp. 478-487, 2010.

[15] N. A. Rahmat, et al., "Economic Load Dispatch with Valve-Point Loading Effect by Using Differential Evolution Immunized Ant Colony Optimization Technique," Australasian Universities Power Engineering Conference AUPEC 2014, Australia, pp. 1-6, 2014.

[16] F. Khodja, et al., "A New Approach ACO for Solving the Compromise Economic and Emission with the Wind Energy,” Energy Procedia, vol. 50, pp. 893-906, 2014.

[17] V. K. Kamboj, et al., "Solution of Non-Convex Economic Load Dispatch Problem for Small-Scale Power Systems using Antlion Optimizer," Neural Computing and Applications, vol. 28, pp. 2181-2192, 2017.

[18] M. Basu, A. Chowdhury, "Cuckoo Search Algorithm for Economic Dispatch,” Energy, vol. 60, pp. 99-108, 2013.

[19] D. N. Vo, et al., "Cuckoo Search Algorithm for Non-Convex Economic Dispatch," IET Generation Transmission and Distribution, vol. 7, pp. 645-654, 2013.

[20] S. Nagaraju, et al., "Economic Load Dispatch Considering Valve Point Loading using Cuckoo Search Algorithm," International journal of Science \& Engineering Development Research, vol. 1, pp. 225-229, 2016.

[21] B. Mallikarjuna, et al., "Economic Load Dispatch Problem With Valve - Point Effect Using A Banary Bat Algorithm," Journal of Electrical Engineering, vol. 14, pp. 67-71, 2014.

[22] B. R. Adarsh, et al., "Economic Dispatch Using Chaotic Bat Algorithm,” Energy, vol. 96, pp. 666-675, 2016.

[23] X. S. Yanga, et al., "Firefly Algorithm for Solving Non-Convex Economic Dispatch Problems with Valve Loading Effect," Applied Soft Computing, vol. 12, pp. 1180-1186, 2012.

[24] D. P. Reddy and M. C. V. Suresh, "Economic Load Dispatch Problem with Valve Point Effect Using Firefly Algorithm,” International Journal of Engineering Sciences \& Research Technology, vol. 4, pp. 557-560, 2015. 
[25] E. D. Manteaw and N. A. Odero, "Combined Economic and Emission Dispatch Solution Using ABC_PSO Hybrid Algorithm with Valve Point Loading Effect," International Journal of Scientific and Research Publications, vol. 2, pp. 1-9, 2012.

[26] Hardiansyah, "Solving Economic Dispatch Problem with Valve-Point Effect using a Modified ABC Algorithm," International Journal of Electrical and Computer Engineering (IJECE), vol. 3(3), pp. 377-385, 2013.

[27] L. Yacine, et al., "Artificial Bee Colony Optimization for Economic Dispatch with Valve Point Effect," Frontiers in Energy, vol. 8, pp. 449-458, 2014.

[28] B. Hadji, et al., "Multi-objective Economic Emission Dispatch Solution Using Dance Bee Colony with Dynamic Step Size," Energy Procedia, vol. 74, pp. 65-76, 2015.

[29] D. Aydın, et al., "Artificial Bee Colony Framework to Non Convex Economic Dispatch Problem with Valve Point Effects: A Case Study," Genetic and Evolutionary Computation Conference (GECCO) 2017, pp. 1131-1318, 2017.

[30] E. Bijami and M. M. Farsangi, "An Improved Adaptive Shuffled Frog Leaping Algorithm to Solve Various Non-smooth Economic Dispatch Problems in Power Systems," Iranian Conference on Intelligent Systems (ICIS), pp. 1-6, 2014.

\section{BIOGRAPHIES OF AUTHORS}

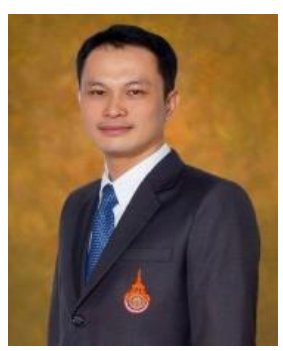

Wanchai Khamsen was born in Lampang, Thailand in 1974. He graduated from Rajamamgla Institute of Technology with a Bachelor's degree in Technical Education in 1997, King Mongkut's Institute of Technology North Bangkok with a Master's degree in Electrical Engineering in 2003 and Ph.D. in Electrical and Computer Engineering of Mahasarakam University. Currently, he is Associate Professor at Faculty of Engineering, Rajamangala University of Technology Lanna Lampang. His research interests include ac choppers, converter systems for improving power quality, economic dispatch and optimization technique.

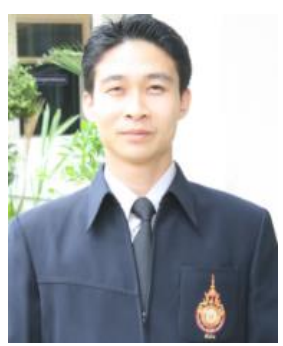

Chiraphon Takeang was born in Chiangrai Thailand in 1978. He received his B.TechED. Degree in Electrical Engineering from the King Mongkut's Institute of Technology North Bangkok in 2002 and the M.Eng. Degree in Electrical Engineering from the Rajamangala University of Technology Lanna in 2014. Currently, he is lecture at Faculty of Engineering, Rajamangala University of Technology Lanna Lampang. His research interests include economic dispatch and optimization technique.

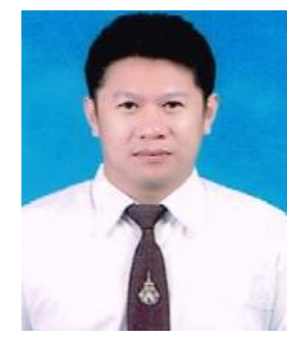

Patiphat Aunban was born in Chiangrai Thailand in 1981. He received his B.Eng. and M.Eng. Degree in Electrical Engineering from the Rajamangala University of Technology Lanna, Thailand in 2005 and 2018. Currently, he is engineering at Chiangmai Municipality, Chiangmai. His research interests include economic dispatch and optimization technique. 